\title{
NEW GLIMPSES ON CONVEX INFINITE OPTIMIZATION DUALITY
}

\author{
M.A. GOBERNA, M.A. LÓPEZ, AND M. VOLLE
}

\begin{abstract}
Given a convex optimization problem $(P)$ in a locally convex topological vector space $X$ with an arbitrary number of constraints, we consider three possible dual problems of $(P)$, namely, the usual Lagrangian dual $(D)$, the perturbational dual $(Q)$, and the surrogate dual $(\Delta)$, the last one recently introduced in a previous paper of the authors [M.A. Goberna, M.A. López, M. Volle, Primal attainment in convex infinite optimization duality, J. Convex Analysis 21, No. 4, 2014, in press]. As shown by simple examples, these dual problems may be all different. This paper provides conditions ensuring that $\inf (P)=\max (D)$, $\inf (P)=\max (Q)$, and $\inf (P)=\max (\Delta)$ (dual equality and existence of dual optimal solutions) in terms of the so-called closedness regarding to a set. Sufficient conditions guaranteeing $\min (P)=\sup (Q)$ (dual equality and existence of primal optimal solutions) are also provided, for the nominal problems and also for their perturbational relatives. The particular cases of convex semi-infinite optimization problems (in which either the number of constraints or the dimension of $X$, but not both, is finite) and linear infinite optimization problems are analyzed. Finally, some applications to the feasibility of convex inequality systems are described.
\end{abstract}

AMS Classif: [2010] Primary 90C25, Secondary 49N15, 46N10.

Keywords: Convex infinite programming, duality.

\section{INTRODUCTION}

Given $m+1$, with $m \geq 1$, convex lower semicontinuous (lsc) proper extended-real-defined functions $f, f_{1}, \ldots, f_{m}$ on a (real) separated locally convex topological vector space $X$ and a non-empty closed convex subset $C$ of $X$, let us consider the convex semi-infinite problem (semi-infinite as the number of constraints is finite but the dimension of $X$ is infinite)

$$
\left(P_{m}\right) \inf _{x} f(x) \text {, s.t. } x \in C, f_{1}(x) \leq 0, \ldots, f_{m}(x) \leq 0 .
$$

Relaxing the inequality constraints, the Lagrangian dual of $\left(P_{m}\right)$ is classically defined as

$$
\left(P_{m}^{\prime}\right) \sup _{\lambda} \inf _{x \in C}\left(f(x)+\sum_{i=1}^{m} \lambda_{i} f_{i}(x)\right) \text {, s.t. } \lambda:=\left(\lambda_{1}, \ldots, \lambda_{m}\right) \in \mathbb{R}_{+}^{m} .
$$

Clearly, some care is necessary in order to give a precise sense to the expression $0 \times(+\infty)$ that may appear in $\left(P_{m}^{\prime}\right)$ formulation. Following Rockafellar 
$[14$, p.24], we may adopt the rule $0 \times(+\infty)=0$. Another possibility is to set $0 \times(+\infty)=+\infty$, a choice made for instance by Zălinescu [15, p.39]. We shall denote by $\left(D_{m}\right)$ and $\left(Q_{m}\right)$ the corresponding versions of $\left(P_{m}^{\prime}\right)$ associated with these rules. It holds that the corresponding optimal values of these problems satisfy

$$
-\infty \leq \sup \left(D_{m}\right) \leq \sup \left(Q_{m}\right) \leq \inf \left(P_{m}\right) \leq+\infty .
$$

Given a non-empty closed convex subset $C$ of $X$ and a family $\left\{f_{t}, t \in T\right\}$ of convex lsc proper functions on $X$, where $T$ is a possibly infinite index set, let us consider now the general convex infinite problem

$$
(P) \inf _{x} f(x) \text {, s.t. } x \in C, f_{t}(x) \leq 0, t \in T,
$$

whose feasible set is $F \cap C$ where

$$
F:=\bigcap_{t \in T}\left[f_{t} \leq 0\right]=\left\{x \in X: f_{t}(x) \leq 0, t \in T\right\} .
$$

The associated Lagrange dual is classically defined as (see, e.g. [3], [5], [7], etc.),

$$
\text { (D) } \sup _{\lambda} \inf _{x \in C}\left(f(x)+\sum_{t \in T} \lambda_{t} f_{t}(x)\right) \text {, s.t. } \lambda:=\left(\lambda_{t}\right)_{t \in T} \in \mathbb{R}_{+}^{(T)},
$$

with $\mathbb{R}_{+}^{(T)}$ denoting the positive cone of the space $\mathbb{R}^{(T)}$ of functions $\lambda: T \rightarrow \mathbb{R}$ whose support $\operatorname{supp} \lambda:=\left\{t \in T: \lambda_{t} \neq 0\right\}$ is finite, and

$$
\sum_{t \in T} \lambda_{t} f_{t}(x):= \begin{cases}0, & \text { if } \lambda=0_{T}, \\ \sum_{t \in \operatorname{supp} \lambda} \lambda_{t} f_{t}(x), & \text { if } \lambda \neq 0_{T}\end{cases}
$$

where $0_{T}$ represents the null-function. It is worth noting that in the case of a finite number of constraints, that is $T=\{1, \ldots, m\}$, the Lagrangian dual $(D)$ coincides with $\left(D_{m}\right)$ while the generalization of $\left(Q_{m}\right)$ is given by (e.g. $[1],[7],[15])$

$$
(Q) \sup _{\lambda} \inf _{x \in C \cap M}\left(f(x)+\sum_{t \in T} \lambda_{t} f_{t}(x)\right) \text {, s.t. } \lambda \in \mathbb{R}_{+}^{(T)},
$$

where $M:=\bigcap_{t \in T} \operatorname{dom} f_{t}$. Observe that if $M \supset C \cap \operatorname{dom} f$, then $(D) \equiv(Q)$.

Finally, replacing the set $\mathbb{R}_{+}^{(T)}$ by $\mathbb{P}(T):=\mathbb{R}_{+}^{(T)} \backslash\left\{0_{T}\right\}$ in the dual problem $(D)$, the following surrogate dual problem $(\Delta)$ was introduced in [7]:

$$
(\Delta) \sup _{\lambda} \inf _{x \in C}\left(f(x)+\sum_{t \in T} \lambda_{t} f_{t}(x)\right) \text {, s.t. } \lambda \in \mathbb{P}(T) \text {. }
$$

One always has the following relations among the optimal value of these problems:

$$
-\infty \leq \sup (\Delta) \leq \sup (D) \leq \sup (Q) \leq \inf (P) \leq+\infty .
$$


The paper is organized as follows. Assuming that $\inf (P)<+\infty$, Section 2 is concerned with the characterization of the so-called strong duality property for the three pairs of dual problems, which respectively accounts for the relations $\inf (P)=\max (D), \inf (P)=\max (Q)$, and $\inf (P)=\max (\Delta)$ (i.e., the optimal values coincide and the dual optimal values are attained) in terms of a property called $w^{*}$-closedness regarding to suitable sets (see [1], [13]). This is the purpose of Theorem 1, the main result in Section 2. Section 3 is devoted to the relation $\min (P)=\sup (\Delta)$ (i.e., we have again dual equality plus attainability of the primal optimal value). Theorem 2 provides sufficient conditions based on the notion of quasicontinuity and recession assumptions. This result improves the one obtained in $[7$, Theorem 4.7] in the sense that we do not assume that $\inf (P)<+\infty$ but only that $\sup (\Delta)<+\infty$. It turns out that the use of this weakened assumption has important consequences. Section 4 shows applications of Theorem 2. In fact, Corollary 1 provides a new general form of the Clark-Duffin's Theorem in terms of the finite intersection property (Corollary 2), while Corollaries 3 and 4 deal with the existence of solutions of convex infinite systems. Section 5 is concerned with the perturbations of the convex infinite problem $(P)$ (Corollary 5), leading us to the characterization of the property $\min (P)=\sup (Q)$ and its perturbational relatives in terms of $w^{*}$-closedness regarding to a set (Theorem 3 and Corollary 6). In this way, Theorems 2 and 3, and Corollaries 5 and 6 complete and improve the results obtained in Section 5 of [7]. In the last Section we apply the previous results to linear infinite optimization problems. Corollaries 7 and 8 provide our most important results in this field.

\section{THE INF-MAX PROPERTY}

We shall start this section with some necessary notation and preliminaries. Given a non-empty subset $A$ of a (real) separated locally convex tvs, we denote by co $A$, cone $A$, aff $A, A^{+}$, and $A^{-}$, the convex hull of $A$, the convex cone generated by $A \cup\left\{0_{X}\right\}$, the smallest linear manifold containing $A$, the positive polar cone of $A$, and the negative polar cone of $A$, respectively. If $A \subset X^{*}$, where $X^{*}$ is the topological dual of $X$, it holds that $A^{++}=A^{--}=$ $\mathrm{cl}^{w^{*}}$ cone $A$. We denote by $C_{\infty}$ the recession cone of the non-empty closed convex set $C$.

Having a function $g: X \rightarrow \overline{\mathbb{R}}:=\mathbb{R} \cup\{ \pm \infty\}$, we denote by epi $g$, epi ${ }_{s} g$, and $g^{*}$ the epigraph, the strict epigraph, and the Legendre-Fenchel conjugate of $g$, respectively. The function $g$ is proper if epi $g \neq \emptyset$ and never takes the value $-\infty$, it is convex if epi $g$ is convex, and it is lower semicontinuous (lsc, in brief) if epi $g$ is closed. We denote by $\Gamma(X)$ the class of lsc proper convex

functions on $X$. The function $\operatorname{cl} \operatorname{cog} g: X \longrightarrow \overline{\mathbb{R}}$ is the lsc convex function such that epi $(\operatorname{cl} \operatorname{co} g)=\operatorname{cl} \operatorname{co}($ epi $g)$. 
The indicator function of $A \subset X$ is represented by $\mathrm{i}_{A}$ (i.e. $\mathrm{i}_{A}(x)=0$ if $x \in A$, and $\mathrm{i}_{A}(x)=+\infty$ if $\left.x \notin A\right)$, and support function of $A$ is the conjugate of its indicator, i.e. $\mathrm{i}_{A}^{*}$. One has $\mathrm{i}_{A}^{*}=\mathrm{i}_{\operatorname{co} A}^{*}=\mathrm{i}_{\operatorname{cl}(\operatorname{co} A)}^{*}$.

Given $g \in \Gamma(X)$, we denote by $g_{\infty}$ its recession function, i.e. the convex function whose epigraph is $(\text { epi } g)_{\infty}$. One has $g_{\infty}:=\mathrm{i}_{\text {dom } g^{*}}^{*}$ (e.g. $[15$, Exercise 2.35]), and

$$
\left[g_{\infty} \leq 0\right]=\left(\operatorname{dom} g^{*}\right)^{-}=\left(\operatorname{conedom} g^{*}\right)^{-},
$$

yielding

$$
\mathrm{cl}^{w^{*}} \text { cone dom } g^{*}=\left[g_{\infty} \leq 0\right]^{-} .
$$

Moreover $\left[g_{\infty} \leq 0\right]=[g \leq \lambda]_{\infty}$ for all $\lambda$ such that $[g \leq \lambda] \neq \emptyset$.

Associated with the dual problems $(\Delta),(D)$ and $(Q)$ we introduce the functions $h, k, \ell: X^{*} \rightarrow \overline{\mathbb{R}}$, respectively defined by

$$
\begin{aligned}
& h:=\inf _{\lambda \in \mathbb{P}(T)}\left(f_{C}+\sum_{t \in T} \lambda_{t} f_{t}\right)^{*}, \\
& k:=\inf _{\lambda \in \mathbb{R}_{+}^{(T)}}\left(f_{C}+\sum_{t \in T} \lambda_{t} f_{t}\right)^{*}, \\
& \ell:=\inf _{\lambda \in \mathbb{R}_{+}^{(T)}}\left(f_{C \cap M}+\sum_{t \in T} \lambda_{t} f_{t}\right)^{*},
\end{aligned}
$$

where $f_{C}:=f+\mathrm{i}_{C}$ and $f_{C \cap M}=f+\mathrm{i}_{C \cap M}$.

The following properties can easily be proved following the same arguments that in [7, Lemmas 3.1 and 3.2] and taking into account the assumptions on $C$ and the functions $f, f_{t}, t \in T$ :

(1) $\ell, k$ and $h$ are convex, and $\ell \leq k \leq h$,

$(2)-\ell\left(0_{X^{*}}\right)=\sup (Q),-k\left(0_{X^{*}}\right)=\sup (D)$, and $-h\left(0_{X^{*}}\right)=\sup (\Delta)$,

(3) $\ell^{*}=k^{*}=h^{*}=f_{C \cap F}$,

(4) $-\ell^{* *}\left(0_{X^{*}}\right)=-k^{* *}\left(0_{X^{*}}\right)=-h^{* *}\left(0_{X^{*}}\right)=\inf (P)$.

The functions $h, k$ and $\ell$ can be improper, possibility which was excluded in [7]. For instance, if $C \cap \operatorname{dom} f=\emptyset$, we obviously have $h=k=\ell \equiv-\infty$. In the following simple example, the functions $f_{C}+\sum_{t \in T} \lambda_{t} f_{t}$ are all proper:

Example 1. Let $X=C=\mathbb{R}^{2}, f(x)=x_{1}, T=\{1\}$, and $f_{1}(x)=\exp \left(x_{2}\right)$. We have $F=\emptyset$, and so $\inf (P)=\inf \left\{x_{1}: \exp \left(x_{2}\right) \leq 0\right\}=+\infty$. Moreover

$$
\sup (\Delta)=\sup _{\lambda>0} \inf _{x \in \mathbb{R}^{2}}\left(x_{1}+\lambda \exp \left(x_{2}\right)\right)=-\infty
$$

and

$$
\sup (D)=\sup (Q)=\sup _{\lambda \geq 0} \inf _{x \in \mathbb{R}^{2}}\left(x_{1}+\lambda \exp \left(x_{2}\right)\right)=-\infty .
$$

For $\lambda>0$, Theorem 2.3.1 [(v),(viii)] in [15] allows us to write

$$
\left(f+\lambda f_{1}\right)^{*}\left(x_{1}^{*}, x_{2}^{*}\right)=\mathrm{i}_{\{1\}}\left(x_{1}^{*}\right)+\lambda \exp ^{*}\left(\lambda^{-1} x_{2}^{*}\right)
$$

where we denote by $\exp ^{*}$ the conjugate of the exponential function exp, i.e.

$$
\exp ^{*}(u)= \begin{cases}+\infty, & u<0 \\ 0, & u=0 \\ u \ln u-u, & u>0\end{cases}
$$


Therefore

$$
\left(f+\lambda f_{1}\right)^{*}\left(x_{1}^{*}, x_{2}^{*}\right)= \begin{cases}+\infty, & x_{1}^{*} \neq 1 \text { or } x_{2}^{*}<0, \\ 0, & x_{1}^{*}=1 \text { and } x_{2}^{*}=0, \\ x_{2}^{*} \ln x_{2}^{*}-x_{2}^{*}-x_{2}^{*} \ln \lambda, & x_{1}^{*}=1 \text { and } x_{2}^{*}>0,\end{cases}
$$

and

$$
h\left(x_{1}^{*}, x_{2}^{*}\right)=\inf _{\lambda>0}\left(f+\lambda f_{1}\right)^{*}\left(x_{1}^{*}, x_{2}^{*}\right)= \begin{cases}+\infty, & x_{1}^{*} \neq 1 \text { or } x_{2}^{*}<0, \\ 0, & x_{1}^{*}=1 \text { and } x_{2}^{*}=0, \\ -\infty, & x_{1}^{*}=1 \text { and } x_{2}^{*}>0 .\end{cases}
$$

We clearly have $h=k=\ell$ and $h^{*}=k^{*}=\ell^{*}=+\infty=f+i_{C \cap F}$. Observe that $h, k, \ell$ are convex but neither proper nor lsc.

We also introduce the sets

$$
\begin{aligned}
\mathfrak{A} & :=\bigcup_{\lambda \in \mathbb{P}(T)} \operatorname{epi}\left(f_{C}+\sum_{t \in T} \lambda_{t} f_{t}\right)^{*}, \\
\mathfrak{B} & :=\bigcup_{\lambda \in \mathbb{R}_{+}^{(T)}} \operatorname{epi}\left(f_{C}+\sum_{t \in T} \lambda_{t} f_{t}\right)^{*}, \\
\mathfrak{C} & :=\bigcup_{\lambda \in \mathbb{R}_{+}^{(T)}} \operatorname{epi}\left(f_{C \cap M}+\sum_{t \in T} \lambda_{t} f_{t}\right)^{*} .
\end{aligned}
$$

It holds that

$$
\operatorname{epi}_{s} h \subset \mathfrak{A} \subset \text { epi } h, \quad \operatorname{epi}_{s} k \subset \mathfrak{B} \subset \text { epi } k, \quad \operatorname{epi}_{s} \ell \subset \mathfrak{C} \subset \text { epi } \ell,
$$

and denoting by $\bar{h}, \bar{k}$ and $\bar{\ell}$ the $w^{*}$-lsc hull of $h, k$ and $\ell$, respectively, we have

$$
\operatorname{epi} \bar{h}=\mathrm{cl}^{w^{*}} \mathfrak{A}, \quad \text { epi } \bar{k}=\mathrm{cl}^{w^{*}} \mathfrak{B}, \quad \text { epi } \bar{\ell}=\mathrm{cl}^{w^{*}} \mathfrak{C} .
$$

Assuming that $C \cap F \cap \operatorname{dom} f \neq \emptyset$ one has, by the convexity of $h, k$ and $\ell$ and (3) above,

$$
\bar{h}=\bar{k}=\bar{\ell}=\left(f_{C \cap F}\right)^{*}=h^{* *}=k^{* *}=\ell^{* *} .
$$

We will need the following notion ([1], see also [13]).

Definition 1. Given two subsets $A, B$ of a topological space, $A$ is said to be closed regarding to $B$ if $B \cap \operatorname{cl} A=B \cap A$.

We are now in a position to state the main result of this section.

Theorem 1. Assume that $\inf (P)<+\infty$. The following assertions are equivalent:

(i) $\mathfrak{A}$ (resp. $\mathfrak{B}$, resp. $\mathfrak{C})$ is $w^{*}$-closed regarding to the set $\left\{0_{X^{*}}\right\} \times \mathbb{R}$.

(ii) $\inf (P)=\max (\Delta)$ (resp. inf $(P)=\max (D)$, resp. inf $(P)=\max (Q))$, including the value $-\infty$.

Proof. We only give the proof relative to $(\Delta)$, the two other ones being similar.

Since $\inf (P)<+\infty$, one has $C \cap F \cap \operatorname{dom} f \neq \emptyset$ and, by $(2.3), \bar{h}=\left(f_{C \cap F}\right)^{*}$. 
Assume first that inf $(P)=-\infty$. By (1.1) we have

$$
\inf _{C}\left(f+\sum_{t \in T} \lambda_{t} f_{t}\right)=-\infty \text { for any } \lambda \in \mathbb{P}(T),
$$

and so, $\inf (P)=-\infty=\max (\Delta)$. On the other hand, $\bar{h}\left(0_{X^{*}}\right)=-\inf (P)=$ $+\infty$ and, by $(2.2)$,

$$
\left(\left\{0_{X^{*}}\right\} \times \mathbb{R}\right) \cap \mathrm{cl}^{w^{*}} \mathfrak{A}=\left(\left\{0_{X^{*}}\right\} \times \mathbb{R}\right) \cap \operatorname{epi} \bar{h}=\emptyset,
$$

implying that $\mathfrak{A}$ is $w^{*}$-closed regarding to $\left\{0_{X^{*}}\right\} \times \mathbb{R}$. So, in the case that $\inf (P)=-\infty$, we have proved that statements $(i)$ and $(i i)$ are simultaneously true.

Assume now that $\alpha:=\inf (P) \in \mathbb{R}$. By (4), (2.2) and (2.3) we have

$$
\left(0_{X^{*}},-\alpha\right) \in \operatorname{epi} h^{* *}=\operatorname{epi} \bar{h}=\operatorname{cl}^{w^{*}} \mathfrak{A} .
$$

Assuming that $(i)$ holds we get $\left(0_{X^{*}},-\alpha\right) \in \mathfrak{A}$, and there exists $\bar{\lambda} \in \mathbb{P}(T)$ such that $\left(f_{C}+\sum_{t \in T} \bar{\lambda}_{t} f_{t}\right)^{*}\left(0_{X^{*}}\right) \leq-\alpha$. This yields

$$
\sup (\Delta) \leq \inf (P)=\alpha \leq \inf _{C}\left\{f_{C}+\sum_{t \in T} \bar{\lambda}_{t} f_{t}\right\} \leq \sup (\Delta)
$$

and $(i i)$ is proved.

Assume now that $(i i)$ holds and let $\left(0_{X^{*}}, r\right) \in \mathrm{cl}^{w^{*}} \mathfrak{A}$. By (4), (2.2) and (2.3), one has $\left(0_{X^{*}}, r\right) \in \operatorname{epi} h^{* *}$ and $-\inf (P)=h^{* *}\left(0_{X^{*}}\right) \leq r$. By $(i i)$, there exists $\bar{\lambda} \in \mathbb{P}(T)$ such that $-\inf (P)=\left(f_{C}+\sum_{t \in T} \bar{\lambda}_{t} f_{t}\right)^{*}\left(0_{X^{*}}\right)$, and we have

$$
\left(0_{X^{*}}, r\right) \in \operatorname{epi}\left(f_{C}+\sum_{t \in T} \bar{\lambda}_{t} f_{t}\right)^{*} \subset \mathfrak{A},
$$

proving that $(i)$ holds.

The next examples compare the characterizations of the inf-max property provided by Theorem 1 with the so-called Slater condition:

$$
\exists \bar{x} \in C \cap \operatorname{dom} f \text { such that } f_{t}(\bar{x})<0 \forall t \in T .
$$

When $T$ is finite, it is known that $-\infty \leq \inf (P)=\max (Q)<+\infty$ whenever the above Slater condition holds ([15, Theorem 2.9.3]).

Example 2. Let $X=C=\mathbb{R}^{2}, f(x)=\exp \left(x_{2}\right), T=\{1\}$, and $f_{1}(x)=$ $x_{1}+\mathrm{i}_{\mathbb{R} \times \mathbb{R}_{+}}(x)$. We have $\inf (P)=\inf \left\{\exp \left(x_{2}\right): x_{1} \leq 0, x_{2} \geq 0\right\}=1$. As the minimum is achieved, we may write $\min (P)=1$, with primal optimal set $S(P)=\mathbb{R}_{-} \times\{0\}$. In order to check the conditions of Theorem 1, we must compute the functions $\left(f+\lambda f_{1}\right)^{*}$ for all $\lambda \geq 0$. If $\lambda>0$, then

$$
\left(f+\lambda f_{1}\right)^{*}\left(x^{*}\right)= \begin{cases}x_{2}^{*} \ln x_{2}^{*}-x_{2}^{*}, & x_{1}^{*}=\lambda, x_{2}^{*}>1 \\ -1, & x_{1}^{*}=\lambda, x_{2}^{*} \leq 1 \\ +\infty, & \text { otherwise }\end{cases}
$$


The above equation remains valid for $\lambda=0$ under the rule $0 \times(+\infty)=+\infty$ (as in $(Q)$ ), but not under the rule $0 \times(+\infty)=0$ (as in $(D)$ ), in which case

$$
\left(f+0 f_{1}\right)^{*}\left(x^{*}\right)= \begin{cases}x_{2}^{*} \ln x_{2}^{*}-x_{2}^{*}, & x_{1}^{*}=0, x_{2}^{*}>0 \\ 0, & x_{1}^{*}=x_{2}^{*}=0, \\ +\infty, & \text { otherwise }\end{cases}
$$

Using again the symbol exp* for the conjugate of the exponential function exp we have

$$
\begin{aligned}
& \mathfrak{A}=\mathbb{R}_{++} \times\left(\operatorname{epi}\left(\exp ^{*}\right)+\mathbb{R}_{+}(-1,0)\right) \\
& \mathfrak{B}=\mathfrak{A} \cup\left(\{0\} \times \operatorname{epi}\left(\exp ^{*}\right)\right) \\
& \mathfrak{C}=\mathbb{R}_{+} \times\left(\operatorname{epi}\left(\exp ^{*}\right)+\mathbb{R}_{+}(-1,0)\right)=\operatorname{cl}^{w^{*}} \mathfrak{A} .
\end{aligned}
$$

The closedness of $\mathfrak{C}$ entails its closedness regarding $\{(0,0)\} \times \mathbb{R}$, while $\mathfrak{A}$ and $\mathfrak{B}$ do not enjoy this property as $\mathfrak{A} \cap(\{(0,0)\} \times \mathbb{R})=\emptyset, \mathfrak{B} \cap(\{(0,0)\} \times \mathbb{R})=$ $\{(0,0, r): r \geq 0\}$, and

$$
\left(\mathrm{cl}^{w^{*}} \mathfrak{A}\right) \cap(\{(0,0)\} \times \mathbb{R})=\left(\mathrm{cl}^{w^{*}} \mathfrak{B}\right) \cap(\{(0,0)\} \times \mathbb{R})=\{(0,0, r): r \geq-1\} .
$$

Thus, by Theorem $1, \inf (P)=\max (Q)$ holds while both $\inf (P)=\max (\Delta)$ and $\inf (P)=\max (D)$ fail. Indeed, $\inf _{\mathbb{R}^{2}}\left\{f+\lambda f_{1}\right\}=-\infty$ for all $\lambda>0$, and

$$
\inf _{\mathbb{R}^{2}}\left\{f+0 f_{1}\right\}= \begin{cases}0, & \text { for }(D) \\ 1, & \text { for }(Q) .\end{cases}
$$

So, $\inf (P)=\max (Q)=1$ (attained for $\lambda=0$ ) while $\sup (D)=\max (D)=0$ (attained for $\lambda=0$ ) and $\sup (\Delta)=-\infty$. Hence, the Slater condition does not guarantee the relation $\inf (P)=\max (D)$, neither $\sup (D)=\sup (Q)$ nor $\sup (D)=\sup (\Delta)$.

Example 3. Let $X=C=\mathbb{R}, f(x)=\exp (x), T=\{1\}$, and $f_{1}(x)=x$. Then, the primal problem is

$$
(P) \inf _{x} \exp (x) \text {, s.t. } x \leq 0,
$$

with associated dual problems

$$
\left.(\Delta) \sup _{\lambda} \inf _{x \in \mathbb{R}}(\exp (x)+\lambda x)\right) \text {, s.t. } \lambda>0,
$$

and

$$
\left.(D) \equiv(Q) \sup _{\lambda} \inf _{x \in \mathbb{R}}(\exp (x)+\lambda x)\right), \text { s.t. } \lambda \geq 0
$$

One has

$$
-\infty=\sup (\Delta)<0=\max (D)=\max (Q)=\inf (P) .
$$

Observe that, for any $\lambda>0$, one has by [15, Theorem 2.3.1(vii)]

$$
\left(f+\lambda f_{1}\right)^{*}\left(x^{*}\right)=f^{*}\left(x^{*}-\lambda\right),
$$


so that epi $\left(f+\lambda f_{1}\right)^{*}=\operatorname{epi}\left(\exp ^{*}\right)+(\lambda, 0)$. Thus,

$$
\mathfrak{A}=\bigcup_{\lambda>0} \operatorname{epi}\left(f+\lambda f_{1}\right)^{*}=\operatorname{epi}\left(\exp ^{*}\right)+(] 0,+\infty[\times\{0\}),
$$

and, analogously, $\mathfrak{B}=\mathfrak{C}=\operatorname{epi}\left(\exp ^{*}\right)+\left(\mathbb{R}_{+} \times\{0\}\right)$. Since

$$
\mathfrak{A} \cap(\{0\} \times \mathbb{R})=\emptyset \neq\{0\} \times \mathbb{R}_{+}=\left(\mathrm{cl}^{w^{*}} \mathfrak{A}\right) \cap(\{0\} \times \mathbb{R}),
$$

$\mathfrak{A}$ is not closed regarding $\{0\} \times \mathbb{R}$ while $\mathfrak{B}=\mathfrak{C}$ is closed and, a fortiori, closed regarding $\{0\} \times \mathbb{R}$. Observe that, once again in this case, Slater condition holds and, however, $\sup (\Delta) \neq \sup (D)$.

Example 4. Let $X=\mathbb{R}, C=[-1,1], f(x)=-x, T=\{1\}$, and $f_{1}(x)=x$ if $x \geq 0, f_{1}(x)=0$ if $x<0$. Now we have

$$
(P) \inf _{x}\{-x, \text { s.t. } x \in[-1,1], x \leq 0\},
$$

with associated dual problems

$$
\begin{gathered}
(D) \equiv(Q) \sup _{\lambda} \inf _{-1 \leq x \leq 1}\left(-x+\lambda f_{1}(x)\right), \text { s.t. } \lambda \geq 0, \\
(\Delta) \sup _{\lambda} \inf _{-1 \leq x \leq 1}\left(-x+\lambda f_{1}(x)\right) \text {, s.t. } \lambda>0 .
\end{gathered}
$$

One has $\inf _{-1 \leq x \leq 1}\left(-x+\lambda f_{1}(x)\right)=0=\inf (P)$ for any $\lambda \geq 1$. Consequently,

$$
\max (\Delta)=\max (D)=\max (Q)=\min (P)=0
$$

In fact, for any $\lambda \geq 0$, one has

$$
\left(f+\lambda f_{1}\right)^{*}\left(x^{*}\right)= \begin{cases}0, & -1 \leq x^{*} \leq \lambda-1, \\ +\infty, & \text { otherwise }\end{cases}
$$

and so $\mathfrak{A}=\mathfrak{B}=\mathfrak{C}=\left[-1,+\infty\left[\times \mathbb{R}_{+}\right.\right.$is closed. However, Slater condition is not satisfied, and this shows that it is sufficient, but not necessary, for having $\inf (P)=\max (Q)<+\infty$.

Example 5. Let $X=C=\mathbb{R}, f(x)=x^{2}, T=\{1\}$, and $f_{1}(x)=x_{+}-1$. Thus, Slater condition holds and we have

$$
\begin{gathered}
(P) \inf _{x} x^{2}, \text { s.t. } x_{+}-1 \leq 0, \\
(\Delta) \sup _{\lambda} \inf _{x \in \mathbb{R}}\left\{x^{2}+\lambda\left(x_{+}-1\right)\right\} \text {, s.t. } \lambda>0,
\end{gathered}
$$

and

$$
(D) \equiv(Q) \sup _{\lambda} \inf _{x \in \mathbb{R}}\left\{x^{2}+\lambda\left(x_{+}-1\right)\right\} \text {, s.t. } \lambda \geq 0 .
$$

By the Moreau-Rockafellar Theorem (see, for instance, [1, Theorem 7.6])

$$
\text { epi }\left(f+\lambda f_{1}\right)^{*}=\operatorname{epi} f^{*}+\operatorname{epi}\left(\lambda f_{1}\right)^{*}=\operatorname{epi} f^{*}+\lambda \text { epi } f_{1}^{*}
$$


for any $\lambda>0$. Setting $\operatorname{pos}(x)=x_{+}, x \in \mathbb{R}$, one has $f_{1}=\operatorname{pos}(\cdot)-1$, $f_{1}^{*}=\operatorname{pos}^{*}(\cdot)+1=\mathrm{i}_{[0,1]}+1$, and so epi $f_{1}^{*}=[0,1] \times[1,+\infty[$. Thus,

$$
\begin{aligned}
\mathfrak{A} & =\bigcup_{\lambda>0} \operatorname{epi}\left(f+\lambda f_{1}\right)^{*} \\
& =\operatorname{epi} f^{*}+\bigcup_{\lambda>0}[0, \lambda] \times[\lambda,+\infty[ \\
& =\left\{\left(x^{*}, r\right): \frac{\left(x^{*}\right)^{2}}{4} \leq r\right\}+\left\{\left(x^{*}, r\right):\left(x^{*}, r\right) \neq(0,0), 0 \leq x^{*} \leq r\right\} \\
& =\left\{\left(x^{*}, r\right): x^{*} \leq 2, \frac{\left(x^{*}\right)^{2}}{4}<r\right\} \cup\left\{\left(x^{*}, r\right): 0<x^{*}-2 \leq r\right\}
\end{aligned}
$$

while

$$
\begin{aligned}
\mathfrak{B}=\mathfrak{C} & =\mathfrak{A} \cup \text { epi } f^{*} \\
& =\left\{\left(x^{*}, r\right): x^{*} \leq 2, \frac{\left(x^{*}\right)^{2}}{4} \leq r\right\} \cup\left\{\left(x^{*}, r\right): 0 \leq x^{*}-2 \leq r\right\} .
\end{aligned}
$$

So, $\mathfrak{B}=\mathfrak{C}$ is closed and equal to epi $\left(f+\mathrm{i}_{]-\infty, 1]}\right)^{*}=\mathrm{cl}^{w^{*}} \mathfrak{A}$. Since

$$
\mathfrak{A} \cap(\{0\} \times \mathbb{R})=\{0\} \times] 0,+\infty\left[\neq\{0\} \times \mathbb{R}_{+}=\left(\mathrm{cl}^{w^{*}} \mathfrak{A}\right) \cap(\{0\} \times \mathbb{R}),\right.
$$

$\mathfrak{A}$ is not closed regarding to $\{0\} \times \mathbb{R}$. This is the reason why $\sup (\Delta)$ is not attained while $\sup (D)=\sup (Q)$ is attained.

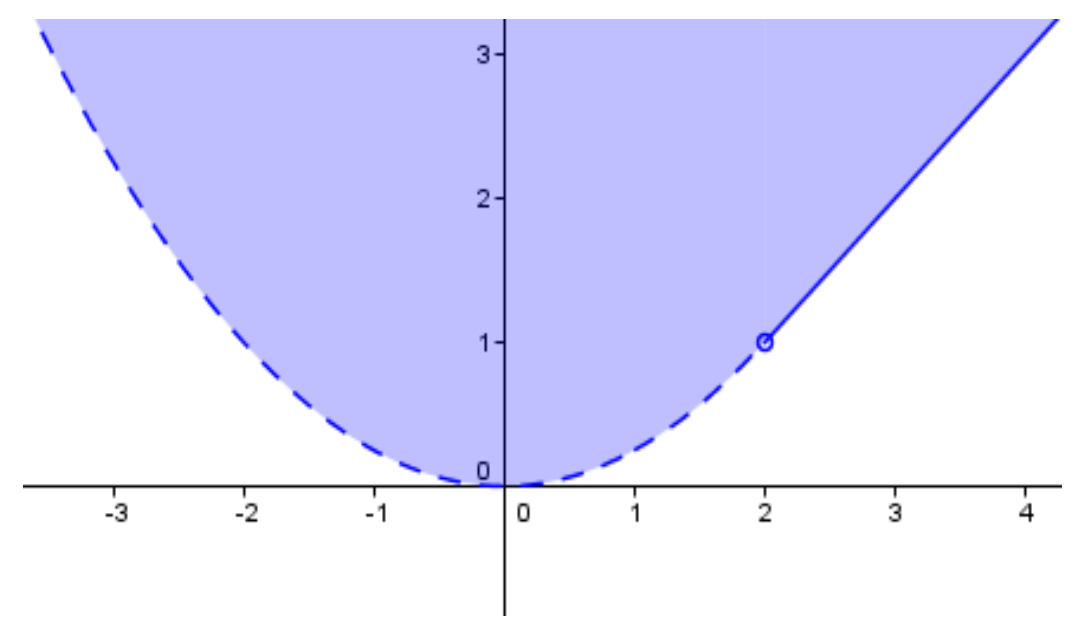

Figure 1. The set $\mathfrak{A}$ in Example 5

\section{ThE MIN-SUP PROPERTY}

With each convex infinite problem

$$
(P) \inf _{x} f(x) \text {, s.t. } x \in C, f_{t}(x) \leq 0, t \in T,
$$


we associate the closed convex cone

$$
\operatorname{rec}(P):=\left[f_{\infty} \leq 0\right] \cap C_{\infty} \cap\left(\bigcap_{t \in T}\left[\left(f_{t}\right)_{\infty} \leq 0\right]\right) .
$$

Obviously, $\operatorname{rec}(P)=\left\{0_{X}\right\}$ if and only if there is no common direction of recession to all the data of $(P)$, namely: $f, C, f_{t}, t \in T$, and it is a linear space if and only if any direction of recession, say $d$, which is common to all the data of $(P)$, if any, is equilibrated in the sense that the opposite direction $-d$ is also common to all the data of $(P)$.

With the convex infinite system formed by the constraints of $(P)$,

$$
\sigma:=\left\{f_{t}(x) \leq 0, t \in T ; x \in C\right\}
$$

is associated the so-called characteristic cone ([2], [3], [6], etc.)

$$
K:=\text { cone }\left\{\operatorname{epi}\left(\mathrm{i}_{C}^{*}\right) \cup\left(\bigcup_{t \in T} \operatorname{epi} f_{t}^{*}\right)\right\}=\operatorname{epi}\left(\mathrm{i}_{C}^{*}\right)+\operatorname{cone}\left(\bigcup_{t \in T} \operatorname{epi} f_{t}^{*}\right)
$$

Now we will make precise some links between $K$ and the epigraph of the function $h$ defined in (2.1). To this end we will just assume that (compare with [5] and [7])

$$
f_{C}+\sum_{t \in T} \lambda_{t} f_{t} \text { is proper for any } \lambda \in \mathbb{P}(T)
$$

Given $\lambda \in \mathbb{P}(T)$ we denote by $\square_{t \in T}\left(\lambda_{t} f_{t}\right)^{*}$ the infimal convolution of the functions $\left(\lambda_{t} f_{t}\right)^{*}, t \in \operatorname{supp} \lambda$, i.e.

$$
\left(\square_{t \in T}\left(\lambda_{t} f_{t}\right)^{*}\right)\left(x^{*}\right)=\inf \left\{\sum_{t \in \operatorname{supp} \lambda}\left(\lambda_{t} f_{t}\right)^{*}\left(x_{t}^{*}\right): \sum_{t \in \operatorname{supp} \lambda} x_{t}^{*}=x^{*}\right\} .
$$

Then, by [15, Theorem 2.8.7],

$$
\left(f_{C}+\sum_{t \in T} \lambda_{t} f_{t}\right)^{*}=\mathrm{cl}^{w^{*}}\left(f^{*} \square \mathrm{i}_{C}^{*} \square\left(\square_{t \in T}\left(\lambda_{t} f_{t}\right)^{*}\right)\right)
$$

Consequently,

$$
\operatorname{epi}\left(f_{C}+\sum_{t \in T} \lambda_{t} f_{t}\right)^{*}=\mathrm{cl}^{w^{*}}\left(\operatorname{epi} f^{*}+\operatorname{epi}\left(\mathrm{i}_{C}^{*}\right)+\sum_{t \in T} \lambda_{t} \text { epi } f_{t}^{*}\right),
$$


so that, by $(2.2)$,

$$
\begin{aligned}
\mathrm{cl}^{w^{*}} \text { epi } h & =\mathrm{cl}^{w^{*}}\left\{\bigcup_{\lambda \in \mathbb{P}(T)} \mathrm{cl}^{w^{*}}\left(\operatorname{epi} f^{*}+\operatorname{epi}\left(\mathrm{i}_{C}^{*}\right)+\sum_{t \in T} \lambda_{t} \text { epi } f_{t}^{*}\right)\right\} \\
& =\mathrm{cl}^{w^{*}}\left\{\operatorname{epi} f^{*}+\operatorname{epi}\left(\mathrm{i}_{C}^{*}\right)+\bigcup_{\lambda \in \mathbb{P}(T)}\left(\sum_{t \in T} \lambda_{t} \text { epi } f_{t}^{*}\right)\right\} \\
& =\operatorname{cl}^{w^{*}}\left\{\operatorname{epi} f^{*}+\operatorname{epi}\left(\mathrm{i}_{C}^{*}\right)+\bigcup_{\lambda \in \mathbb{R}_{+}^{(T)}}\left(\sum_{t \in T} \lambda_{t} \text { epi } f_{t}^{*}\right)\right\} \\
& =\mathrm{cl}^{w^{*}}\left(\operatorname{epi} f^{*}+K\right) .
\end{aligned}
$$

We thus have

$$
\mathrm{cl}^{w^{*}} \text { cone epi } h=\mathrm{cl}^{w^{*}} \text { cone }\left(\mathrm{cl}^{w^{*}} \text { epi } h\right)=\mathrm{cl}^{w^{*}} \text { cone }\left(\text { epi } f^{*}+K\right)
$$

and, finally,

$$
\mathrm{cl}^{w^{*}} \text { cone epi } h=\mathrm{cl}^{w^{*}}\left(K+\text { cone epi } f^{*}\right) \text {. }
$$

Denoting by $\Pi$ the projection of $X^{*} \times \mathbb{R}$ onto $X^{*}$ one has, according to $(3.2)$,

$$
\begin{gathered}
\mathrm{cl}^{w^{*}} \text { cone dom } h=\mathrm{cl}^{w^{*}} \text { cone } \Pi(\text { epi } h)=\mathrm{cl}^{w^{*}} \Pi(\text { cone epi } h) \\
=\mathrm{cl}^{w^{*}} \Pi\left(\mathrm{cl}^{w^{*}} \text { cone epi } h\right)=\mathrm{cl}^{w^{*}} \Pi\left(K+\text { cone epi } f^{*}\right)
\end{gathered}
$$

Using the definition of $K$ we get the key relation

$$
\mathrm{cl}^{w^{*}} \text { cone } \operatorname{dom} h=\mathrm{cl}^{w^{*}}\left(b(C)+\operatorname{cone}\left(\bigcup_{t \in T} \operatorname{dom} f_{t}^{*}\right)+\operatorname{conedom} f^{*}\right),
$$

where $b(C):=\operatorname{dom}\left(\mathrm{i}_{C}^{*}\right)$ denotes the barrier cone of $C$.

Since the condition

$$
\mathrm{cl}^{w^{*}} \text { cone dom } h \text { is a linear space }
$$

will be of crucial importance in the sequel, we summarize below some equivalent reformulations of (3.4). To this aim we need the following equivalence whose simple proof is omitted: Having a linear space $U$ and a function $g: U \rightarrow \overline{\mathbb{R}}$ it holds that

$$
(\operatorname{dom} g) \times \mathbb{R}=(\text { epi } g)-\left\{0_{U}\right\} \times \mathbb{R}_{+} .
$$

Proposition 1. Assume that (3.1) holds. Then, each of the following statements is equivalent to (3.4):

(i) $\operatorname{rec}(P)$ is a linear space.

(ii) $\mathrm{cl}^{w^{*}}\left(b(C)+\right.$ cone $\left(\bigcup_{t \in T} \operatorname{dom} f_{t}^{*}\right)+$ cone dom $\left.f^{*}\right)$ is a linear space.

(iii) $\mathrm{cl}^{w^{*}}\left(K+\right.$ cone epi $\left.f^{*}-\left\{0_{X^{*}}\right\} \times \mathbb{R}_{+}\right)$is a linear space. 
$(i v) \mathrm{cl}^{w^{*}}\left(K \cup\right.$ epi $\left.f^{*} \cup\left\{\left(0_{X^{*}},-1\right)\right\}\right)$ is a linear space.

$(v) \mathrm{cl}^{w^{*}}\left(b(C) \times \mathbb{R}+\right.$ cone $\left(\bigcup_{t \in T}\right.$ epi $\left.f_{t}^{*}\right)+$ cone epi $\left.f^{*}\right)$ is a linear space.

Proof: By taking the negative polar cone we obtain that $(i) \Leftrightarrow(i i)$. By (3.2) and (3.5) one has

$$
\begin{aligned}
\left(\mathrm{cl}^{w^{*}} \text { cone dom } h\right) \times \mathbb{R} & =\mathrm{cl}^{w^{*}} \text { cone }\left(\text { epi } h-\left\{0_{X^{*}}\right\} \times \mathbb{R}_{+}\right) \\
& =\mathrm{cl}^{w^{*}}\left(\mathrm{cl}^{w^{*}} \text { cone epi } h-\left\{0_{X^{*}}\right\} \times \mathbb{R}_{+}\right) \\
& =\operatorname{cl}^{w^{*}}\left(K+\text { cone epi } f^{*}-\left\{0_{X^{*}}\right\} \times \mathbb{R}_{+}\right) .
\end{aligned}
$$

It follows that $(3.4) \Leftrightarrow(i i i)$. Since $K$ is a cone, one has

$$
K+\text { cone epi } f^{*}-\left\{0_{X^{*}}\right\} \times \mathbb{R}_{+}=\text {cone }\left(K \cup \text { epi } f^{*} \cup\left\{\left(0_{X^{*}},-1\right)\right\}\right) .
$$

We thus have $(i i i) \Leftrightarrow(i v)$. By $(3.5)$ one has epi $\left(\mathrm{i}_{C}^{*}\right)-\left\{0_{X^{*}}\right\} \times \mathbb{R}_{+}=b(C) \times \mathbb{R}$. From the very definition of $K$, it follows that $($ iii $) \Leftrightarrow(v)$.

3.1. Quasicontinuity and subdifferentiability. We denote by $w$ (respectively, $\tau^{*}$ ) the weak topology on $X$ (respectively, the Mackey topology on $X^{*}$ ). Following [9] and [10], a convex function $g: X^{*} \rightarrow \overline{\mathbb{R}}$ is said to be $\tau^{*}$ quasicontinuous when the affine hull of $\operatorname{dom} g$, aff $\operatorname{dom} g$, is $w^{*}$-closed and of finite codimension, and the restriction of $g$ to the relative interior of $\operatorname{dom} g$, say $\mathrm{ri}^{\tau^{*}} \operatorname{dom} g$, is continuous with respect to the topology induced by $\tau^{*}$.

If $g$ is $w^{*}$-lsc and proper, one has ([11, Theorem 7.7.6]):

$$
g \text { is } \tau^{*} \text {-quasicontinuous } \Leftrightarrow g^{*} \text { is } w \text {-inf-locally-compact, }
$$

meaning that for each $r \in \mathbb{R}$, the sublevel set $\left[g^{*} \leq r\right]$ is $w$-locally-compact.

Any extended real-valued convex function which is majorized by a $\tau^{*}$ quasicontinuous convex function is $\tau^{*}$-quasicontinuous too [12, Theorem 2.4]. Accordingly, the convex function $h$ defined in (2.1) is $\tau^{*}$-quasicontinuous whenever there exists $\bar{\lambda} \in \mathbb{P}(T)$ such that $f_{C}+\sum_{t \in T} \bar{\lambda}_{t} f_{t}$ is $w$-inf-locallycompact (this fact is observed in [7, p.11]). Such a condition is in particular fulfilled when $C$ is $w$-locally-compact, e.g. when $X$ is finite dimensional.

We will use the following subdifferentiability criterion [12, Theorem 3.3].

Lemma 1. Let $g: X^{*} \rightarrow \overline{\mathbb{R}}$ be convex and $\tau^{*}$-quasicontinuous. Assume that $g\left(0_{X^{*}}\right)>-\infty$ and $\mathrm{cl}^{w^{*}}$ cone dom $g$ is a linear space. Then, $\partial g\left(0_{X^{*}}\right)$ is the sum of a non-empty $w$-compact convex set and a finite dimensional linear space.

3.2. The main result. Remember that by $S(P)$ we denote the optimal solution set of the convex infinite problem

$$
(P) \inf _{x} f(x) \text {, s.t. } x \in C, f_{t}(x) \leq 0, t \in T,
$$


and recall also the formulation of the surrogate dual $(\Delta)$ of $(P)$ :

$$
(\Delta) \sup _{\lambda} \inf _{C}\left(f+\sum_{t \in T} \lambda_{t} f_{t}\right) \text {, s.t. } \lambda \in \mathbb{P}(T) .
$$

Theorem 2. Assume that the following assumptions are fulfilled:

$$
\begin{gathered}
\qquad \sup (\Delta)<+\infty \\
\exists \bar{\lambda} \in \mathbb{R}_{+}^{(T)} \text { such that } f_{C}+\sum_{t \in T} \bar{\lambda}_{t} f_{t} \text { is w-inf-locally-compact, }
\end{gathered}
$$

and

$$
\operatorname{rec}(P) \text { is a linear space. }
$$

Then, $\min (P)=\sup (\Delta) \in \mathbb{R}$, and $S(P)$ is the sum of a non-empty $w$ compact convex set and a finite dimensional linear space.

Proof: Let us apply Lemma 1 to $g=h$. By (3.6) one has $h\left(0_{X^{*}}\right)>-\infty$. By (3.7), $h$ is $\tau^{*}$-quasicontinuous and, by (3.3), (3.8) and the equivalence $(i) \Leftrightarrow($ ii $)$ in Proposition $1, \mathrm{cl}^{w^{*}}$ cone dom $h$ is a linear space. By Lemma $1, \partial h\left(0_{X^{*}}\right)$ is the sum of a non-empty $w$-compact convex set and a finite dimensional linear space. Now $x \in \partial h\left(0_{X^{*}}\right)$ means that $-h\left(0_{X^{*}}\right)=h^{*}(x)=$ $f_{C \cap F}(x) \in \mathbb{R}$. In other words, $x$ is feasible for $(P)$ and

$$
\inf (P) \geq \sup (\Delta)=h^{*}(x)=f(x) \geq \inf (P) .
$$

We thus have $\min (P)=\sup (\Delta) \in \mathbb{R}$ and $\partial h\left(0_{X^{*}}\right) \subset S(P)$. To complete the proof, take $\bar{x} \in S(P)$ and write

$$
+\infty>\sup (\Delta)=-h^{*}\left(0_{X^{*}}\right)=\min (P)=f(\bar{x})=f_{C \cap F}(\bar{x})=h^{*}(\bar{x}),
$$

i.e., $h^{*}(\bar{x})+h\left(0_{X^{*}}\right)=0=\left\langle 0_{X^{*}}, \bar{x}\right\rangle$, entailing $\bar{x} \in \partial h\left(0_{X^{*}}\right)$.

Let us revisit the examples of Section 2, where $X$ is finite dimensional and $\sup (\Delta)<+\infty$, so that Theorem 2 applies whenever rec $(P)$ is a linear space. This is the case of Examples 4 and 5, where $\operatorname{rec}(P)=\{0\}$, with $\sup (\Delta)$ attained in Example 4 but not in Example 5. Observe that, in Example 2, $\operatorname{rec}(P)=\mathbb{R}_{-} \times\{0\}$, with $\inf (P)=1 \neq-\infty=\sup (\Delta)$, while, in Example $3, \operatorname{rec}(P)=\mathbb{R}_{-}$, with $\inf (P)=0 \neq-\infty=\sup (\Delta)$.

Remark 1. The same conclusion is obtained in [7, Theorems 4.7 and 4.8] replacing condition (3.6) by the stronger assumption that inf $(P)<+\infty$.

Remark 2. In the case that $\sup (\Delta)=+\infty$, all the problems $(P),(D)$ and $(Q)$ share the same value.

Now provide a new version of the famous Clark-Duffin Theorem for semiinfinite optimization with $T$ finite. We are concerned with the problems

$$
\left(P_{m}\right) \inf _{x} f(x), \text { s.t. } x \in C, f_{1}(x) \leq 0, \ldots, f_{m}(x) \leq 0,
$$




$$
\left(Q_{m}\right) \sup _{\lambda} \inf _{C}\left(f+\sum_{i=1}^{m} \lambda_{i} f_{i}\right) \text {, s.t. }\left(\lambda_{1}, \ldots, \lambda_{m}\right) \in \mathbb{R}_{+}^{m},
$$

with the rule $0 \times(+\infty)=+\infty$,

$$
\left(D_{m}\right) \sup _{\lambda} \inf _{C}\left(f+\sum_{i=1}^{m} \lambda_{i} f_{i}\right) \text {, s.t. }\left(\lambda_{1}, \ldots, \lambda_{m}\right) \in \mathbb{R}_{+}^{m},
$$

with the rule $0 \times(+\infty)=0$, and

$$
\left(\Delta_{m}\right) \sup _{\lambda} \inf _{C}\left(f+\sum_{i=1}^{m} \lambda_{i} f_{i}\right), \text { s.t. }\left(\lambda_{1}, \ldots, \lambda_{m}\right) \in \mathbb{R}_{+}^{m} \backslash\left\{0_{\mathbb{R}^{m}}\right\},
$$

where $X$ is a locally convex separated tvs, $C$ a non-empty closed convex subset of $X$ and $f, f_{1}, \ldots, f_{m} \in \Gamma(X)$. The next result is to be compared with [8, Theorem 5.1] and [4, Theorem 3.1].

Corollary 1. Assume that $\sup \left(\Delta_{m}\right)<+\infty$, that there exists $\bar{\lambda} \in \mathbb{R}_{+}^{m}$ such that $f_{C}+\sum_{i=1}^{m} \bar{\lambda}_{i} f_{i}$ is $w$-inf-locally-compact, with the rule $0 \times(+\infty)=0$, and that $\operatorname{rec}\left(P_{m}\right)$ is a linear space. Then,

$$
\sup \left(\Delta_{m}\right)=\sup \left(D_{m}\right)=\sup \left(Q_{m}\right)=\min \left(P_{m}\right) \in \mathbb{R}
$$

and $S\left(P_{m}\right)$ is the sum of a non-empty $w$-compact convex set and a finite dimensional linear space.

Remark 3. If $X$ is finite dimensional, the second assumption in the statement of Corollary 1 is superfluous.

\section{Applications}

4.1. The finite intersection property. Recall that a family $\left\{C_{t}, t \in T\right\}$ of sets of a topological space is said to have the finite-intersection property if every finite subfamily has non-empty intersection. As a substitute of compactness we have the following result:

Corollary 2. Let $\left\{C_{t}, t \in T\right\}$ be a family of closed convex subsets of a locally convex separated tvs having the finite-intersection property. Moreover, assume the existence of $t_{1}, \ldots, t_{m} \in T$ such that $\bigcap_{i=1}^{m} C_{t_{i}}$ is $w$-locally-compact and that $\bigcap_{t \in T}\left(C_{t}\right)_{\infty}$ is a linear space. Then $\bigcap_{t \in T} C_{t}$ is the sum of a nonempty w-compact convex set and a finite dimensional linear space.

Proof Apply Theorem 2 with $C=X, f \equiv 0$, and $f_{t}=\mathrm{i}_{C_{t}}, t \in T$, observing that $S(P)=\bigcap_{t \in T} C_{t}, \operatorname{rec}(P)=\bigcap_{t \in T}\left(C_{t}\right)_{\infty}$, and $\sup (\Delta)<$ $+\infty$ amounts to say that the family $\left\{C_{t}, t \in T\right\}$ has the finite-intersection property.

Remark 4. Taking $C=X=\mathbb{R}, f \equiv 0$, and $f_{t}=\mathrm{i}_{[t,+\infty[}, t>0$, in Theorem 2 , we get $M=\emptyset$ and, since the family $\{[t,+\infty[, t>0\}$ has the finiteintersection property, one gets

$$
\max (\Delta)=\max (D)=0<+\infty=\sup (Q)=\inf (P) .
$$


Since $\operatorname{rec}(P)=[0,+\infty[$ is not a linear space, the assumption $(3.8)$ in Theorem 2 is not satisfied.

4.2. Convex infinite systems. In this section we again apply Theorem 2 in the case that $f \equiv 0$. We denote by $\left(P_{0}\right)$ the corresponding convex infinite problem, and by

$$
\sigma:=\left\{f_{t}(x) \leq 0, t \in T ; x \in C\right\},
$$

the general infinite convex system associated with the constraints of $\left(P_{0}\right)$, whereas $K$ is the characteristic cone of $\sigma$. The feasible set $C \cap F$ of $\sigma$ coincides with $S\left(P_{0}\right)$. It may be empty even if we assume that $\sup \left(\Delta_{0}\right)<+\infty$ (see Remark 4).

The function $h_{0}$ associated with $\left(P_{0}\right)$ is

$$
h_{0}=\inf _{\lambda \in \mathbb{P}(T)}\left(\mathrm{i}_{C}+\sum_{t \in T} \lambda_{t} f_{t}\right)^{*} .
$$

Assuming that

$$
\mathrm{i}_{C}+\sum_{t \in T} \lambda_{t} f_{t} \text { is proper for any } \lambda \in \mathbb{P}(T),
$$

which is the counterpart of (3.1) and it is weaker than $\sup \left(\Delta_{0}\right)<+\infty$, it holds that

$$
\mathrm{cl}^{w^{*}} \text { epi } h_{0}=\mathrm{cl}^{w^{*}} K
$$

and, recalling (3.3),

$$
\mathrm{cl}^{w^{*}} \text { cone dom } h_{0}=\mathrm{cl}^{w^{*}}\left(b(C)+\operatorname{cone}\left(\bigcup_{t \in T} \operatorname{dom} f_{t}^{*}\right)\right) .
$$

Let us define the recession cone associated with $\sigma$ by

$$
\operatorname{rec}(\sigma):=\operatorname{rec}\left(P_{0}\right)=C_{\infty} \cap\left(\bigcap_{t \in T}\left[\left(f_{t}\right)_{\infty} \leq 0\right]\right) .
$$

Assuming that (4.1) holds, the following assertions are equivalent (see Proposition 1):

$\left(i_{0}\right) \quad \operatorname{rec}(\sigma)$ is a linear space,

$\left(i i_{0}\right) \quad \mathrm{cl}^{w^{*}}\left(b(C)+\right.$ cone $\left.\left(\bigcup_{t \in T} \operatorname{dom} f_{t}^{*}\right)\right)$ is a linear space,

(iii $)_{0} \quad \mathrm{cl}^{w^{*}}\left(K-\left\{0_{X^{*}}\right\} \times \mathbb{R}_{+}\right)$is a linear space,

$\left(i v_{0}\right) \quad \mathrm{cl}^{w^{*}}$ cone $\left(K \cup\left\{\left(0_{X^{*}},-1\right)\right\}\right)$ is a linear space,

$\left(v_{0}\right) \quad \mathrm{cl}^{w^{*}}\left(b(C) \times \mathbb{R}+\right.$ cone $\left(\bigcup_{t \in T}\right.$ epi $\left.\left.f_{t}^{*}\right)\right)$ is a linear space.

We are now in a position to state a generalization of Fan's Theorem in general locally convex separated tvs: 
Corollary 3. Assume that

$\exists \bar{\lambda} \in \mathbb{R}_{+}^{m}$ such that $\mathrm{i}_{C}+\sum_{t \in T} \bar{\lambda}_{t} f_{t}$ is w-inf-locally-compact,

and that

$$
\operatorname{rec}(\sigma) \text { is a linear space. }
$$

Then, the infinite convex system $\sigma$ is consistent if and only if

$$
\inf _{C} \sum_{t \in T} \lambda_{t} f_{t} \leq 0 \text { for any } \lambda \in \mathbb{P}(T) \text {. }
$$

Proof: Necessity is obvious. Sufficiency comes from Theorem 2 by taking $f \equiv 0$.

Remark 5. With the same assumptions, statement (4.4) in Corollary 3 is equivalent to

$$
\forall \lambda \in \mathbb{R}_{+}^{(T)}, \exists x_{\lambda} \in C \text { such that } \sum_{t \in T} \lambda_{t} f_{t}\left(x_{\lambda}\right) \leq 0
$$

that appears in [2, Theorem 3.5].

In [2, Theorem 3.5] it is assumed that either $K$ is $w^{*}$-closed or $K$ is solid if $X$ is infinite dimensional, and $\operatorname{rec}(\sigma)=\left\{0_{X^{*}}\right\}$. We now provide an example where none of these two conditions is satisfied while Corollary 3 does work.

Example 6. Let $X$ be a reflexive Banach space whose open (respectively, closed) unit dual ball is represented by $\mathbb{B}^{*}$ (resp., $\overline{\mathbb{B}}^{*}$ ). Notice that the topology $\tau^{*}$ coincides with the dual norm topology. Given $a \in X, a \neq 0_{X}$, let us set $H:=\{a\}^{\perp}$ and consider

$$
D:=H \cap \overline{\mathbb{B}}^{*} .
$$

It holds that cone $D=$ aff $D=H$, a closed hyperplane, and $0_{X^{*}} \in \operatorname{ri} D=$ $H \cap \mathbb{B}^{*}$. Setting $f_{t}:=\mathrm{i}_{D}^{*}-\frac{1}{t}, t>0$, we get a family of functions in $\Gamma(X)$ having the same recession cone, namely,

$$
\left[\left(f_{t}\right)_{\infty} \leq 0\right]=\left[\mathrm{i}_{D}^{*} \leq 0\right]=H^{\perp}=\mathbb{R}\{a\} \text {, for all } t>0 .
$$

Since $f_{t}^{*}=\mathrm{i}_{D}+\frac{1}{t}$ is $\tau^{*}$-quasicontinuous, any $f_{t}$ is $w$-inf-locally-compact. Consequently, the system

$$
\sigma:=\left\{f_{t}(x) \leq 0, t>0\right\}
$$

satisfies the assumptions of our Corollary 3. However,

$$
K=\text { cone }\left(\bigcup_{t>0} \operatorname{epi} f_{t}^{*}\right)=(H \times] 0,+\infty[) \cup\left\{\left(0_{X^{*}}, 0\right)\right\}
$$

is not $w^{*}$-closed, $K \subset H \times \mathbb{R}$ is not solid, and $\operatorname{rec}(\sigma)=\mathbb{R}\{a\}$ is not $\left\{\left(0_{X^{*}}, 0\right)\right\}$. Consequently, the assumptions of $[2$, Theorem 3.5] are not satisfied. 
Given $m \geq 1, t_{1}, \ldots, t_{m} \in T$, and $\varepsilon>0$, let us consider the system

$$
\sigma\left(t_{1}, \ldots, t_{m}, \varepsilon\right):=\left\{f_{t_{i}}(x) \leq \varepsilon, i=1, \ldots, m, x \in C\right\} .
$$

Corollary 4. Assume that (4.2) and (4.3) hold. Then the convex infinite system $\sigma$ is consistent if and only if all the semi-infinite systems $\sigma\left(t_{1}, \ldots, t_{m}, \varepsilon\right), m \geq 1, t_{1}, \ldots, t_{m} \in T, \varepsilon>0$, are consistent.

Proof: Necessity is obvious; now we show the sufficiency. Applying Corollary 3, we have just to verify that (4.4) holds. So, let $\lambda \in \mathbb{P}(T)$ and $\operatorname{supp} \lambda=\left\{t_{1}, \ldots, t_{m}\right\}$. For any $\alpha>0$ there exists $\bar{x} \in C$ such that

$$
f_{t_{i}}(\bar{x}) \leq \frac{\alpha}{\sum_{j=1}^{m} \lambda_{j}}, i=1, \ldots, m \text {. }
$$

We thus have

$$
\sum_{t \in T} \lambda_{t} f_{t}(\bar{x})=\sum_{i=1}^{m} \lambda_{t_{i}} f_{t_{i}}(\bar{x}) \leq \alpha
$$

Since $\alpha>0$ is arbitrary, we have that (4.4) holds.

Remark 6. Every time, when the conditions of Corollaries 3 and 4 are fulfilled, then the solution set of the convex infinite system $\sigma$ is the sum of a non-empty $w$-compact convex set and a finite dimensional linear space.

\section{Perturbational approach}

Having $\mu=\left(\mu_{t}\right)_{t \in T} \in \mathbb{R}^{T}$, we consider the parametric convex infinite problem

$$
\left(P^{\mu}\right) \inf _{x} f(x) \text {, s.t. } x \in C, f_{t}(x) \leq-\mu_{t}, t \in T,
$$

where $f, f_{t}, t \in T$, are proper convex functions defined on the locally convex separated tvs $X$, and $C \subset X$ is a non-empty convex set. Let us observe that all these problems have the same recession cone:

$$
\operatorname{rec}\left(P^{\mu}\right)=\operatorname{rec}\left(P^{0_{T}}\right)=\operatorname{rec}(P) .
$$

Considering the associated dual problems

$$
\begin{aligned}
& \left(D^{\mu}\right) \sup _{\lambda}\left\{\sum_{t \in T} \lambda_{t} \mu_{t}+\inf _{C}\left(f+\sum_{t \in T} \lambda_{t} f_{t}\right)\right\}, \text { s.t. } \lambda \in \mathbb{R}_{+}^{(T)}, \\
& \left(\Delta^{\mu}\right) \sup _{\lambda}\left\{\sum_{t \in T} \lambda_{t} \mu_{t}+\inf _{C}\left(f+\sum_{t \in T} \lambda_{t} f_{t}\right)\right\}, \text { s.t. } \lambda \in \mathbb{P}(T),
\end{aligned}
$$

we can thus state, applying Theorem 2 :

Corollary 5. Assume that (3.7) and (3.8) hold. For any $\mu \in \mathbb{R}^{T}$ we have either

$$
\min \left(P^{\mu}\right)=\sup \left(D^{\mu}\right)=\sup \left(\Delta^{\mu}\right) \in \mathbb{R},
$$

or

$$
\inf \left(P^{\mu}\right)=\sup \left(D^{\mu}\right)=\sup \left(\Delta^{\mu}\right)=+\infty
$$


By using the value function $v: \mathbb{R}^{T} \rightarrow \overline{\mathbb{R}}$,

$$
v(\mu):=\inf \left(P^{\mu}\right)
$$

we can develop in a natural way the classical perturbational duality theory for convex infinite problems (see, e.g. [1], [15]) by computing the conjugate of $v$, namely,

$$
-v^{*}(\lambda)= \begin{cases}\inf _{C \cap M}\left(f+\sum_{t \in T} \lambda_{t} f_{t}\right), & \text { if } \lambda \in \mathbb{R}_{+}^{(T)}, \\ -\infty, & \text { if } \lambda \in \mathbb{R}^{(T)} \backslash \mathbb{R}_{+}^{(T)},\end{cases}
$$

and defining the perturbational dual of $\left(P^{\mu}\right)$ as

$$
\left(Q^{\mu}\right) \sup _{\lambda}\left\{\sum_{t \in T} \lambda_{t} \mu_{t}+\inf _{C \cap M}\left(f+\sum_{t \in T} \lambda_{t} f_{t}\right)\right\} \text {, s.t. } \lambda \in \mathbb{R}_{+}^{(T)} .
$$

We observe that $\left(Q^{0_{T}}\right)$ coincides with the problem $(Q)$ defined in Section 1 .

One has, in general, the following well-known properties:

a) $-\infty \leq \sup \left(\Delta^{\mu}\right) \leq \sup \left(D^{\mu}\right) \leq \sup \left(Q^{\mu}\right)=v^{* *}(\mu) \leq v(\mu)=\inf \left(P^{\mu}\right) \leq$ $+\infty$

b) $E:=\bigcup_{x \in C \cap M \cap \operatorname{dom} f}\left\{\left(\left(f_{t}(x)\right)_{t \in T}, f(x)\right)\right\}+\mathbb{R}_{+}^{T} \times \mathbb{R}_{+}$is convex,

c) $v$ is convex,

d) $\operatorname{epi}_{s} v \subset \widehat{E}:=\left\{(\mu, r) \in \mathbb{R}^{T} \times \mathbb{R}:(-\mu, r) \in E\right\} \subset$ epi $v$, and

e) epi $\bar{v}=\operatorname{cl}$ epi $v=\operatorname{cl} \widehat{E}$.

Observe that all these properties are true just assuming the convexity of the data of $(P): f, C, f_{t}, t \in T$.

Theorem 3. Assume that $f, f_{t}: X \rightarrow \mathbb{R} \cup\{+\infty\}$ are proper convex and $C$ is a non-empty convex subset of the locally convex tvs $X$ such that

$$
\exists \bar{\lambda} \in \mathbb{R}_{+}^{(T)} \text { such that } \inf _{C \cap M}\left(f+\sum_{t \in T} \bar{\lambda}_{t} f_{t}\right) \neq-\infty .
$$

Then, for any $\mu \in \mathbb{R}^{T}$, the following statements are equivalent:

(i) $\min \left(P^{\mu}\right)=\sup \left(Q^{\mu}\right) \in \mathbb{R}$ or $\sup \left(Q^{\mu}\right)=+\infty$.

(ii) $E$ is closed regarding to $\{-\mu\} \times \mathbb{R}$.

Proof: By (5.1) and (5.2) one has $v^{*}(\bar{\lambda})<+\infty$ and so, dom $v^{*} \neq \emptyset$. Since $v$ is convex, $\bar{v}=v^{* *}$ (either $v$ is proper or $+\infty=v^{* *}=\bar{v}=v$ ).

Let us begin with the case that $\sup \left(Q^{\mu}\right)=+\infty$. Then $\bar{v}(\mu)=+\infty$ and

$$
\emptyset=(\{\mu\} \times \mathbb{R}) \cap \operatorname{epi} \bar{v}=(\{\mu\} \times \mathbb{R}) \cap \operatorname{cl} \widehat{E} .
$$

So, $\widehat{E}$ is closed regarding to $\{\mu\} \times \mathbb{R}$ and, equivalently, $E$ is closed regarding to $\{-\mu\} \times \mathbb{R}$. Thus, if $\sup \left(Q^{\mu}\right)=+\infty$, the statements $(i)$ and $(i i)$ are simultaneously satisfied. 
Assume now that $\beta:=\sup \left(Q^{\mu}\right)<+\infty$. By (5.2) we have $\beta \in \mathbb{R}$ and so $(\mu, \beta) \in \operatorname{cl}$ epi $v=\operatorname{cl} \widehat{E}$, that is

$$
(-\mu, \beta) \in \operatorname{cl} E \text {. }
$$

Assume that $(i)$ holds and let $(-\mu, r) \in \operatorname{cl} E$, so that $\bar{v}(\mu)=\beta \leq r$. Taking $\bar{x} \in S\left(P^{\mu}\right)$ we get $\bar{x} \in C \cap M \cap \operatorname{dom} f, f_{t}(\bar{x}) \leq-\mu_{t}, t \in T$, and $f(\bar{x})=\beta \leq r$. So,

$$
(-\mu, r) \in\left\{\left(\left(f_{t}(\bar{x})\right)_{t \in T}, f(\bar{x})\right)\right\}+\mathbb{R}_{+}^{(T)} \times \mathbb{R}_{+} \subset E,
$$

and (ii) holds.

Conversely, assume that (ii) holds. By (5.3) we thus have $(-\mu, r) \in E$, and there exists $\bar{x} \in C \cap M \cap \operatorname{dom} f$ such that

$$
f_{t}(\bar{x}) \leq-\mu_{t}, t \in T, f(\bar{x}) \leq \beta \leq \inf \left(P^{\mu}\right) .
$$

Since $\bar{x}$ is feasible for $\left(P^{\mu}\right)$, we obtain $(i)$.

This section ends with an application of Theorem 3 to the convex system

$$
\sigma:=\left\{f_{t}(x) \leq 0, t \in T ; x \in C\right\},
$$

where $f_{t}: X \rightarrow \mathbb{R} \cup\{+\infty\}, t \in T$, are proper convex and $C$ is a non-empty convex subset of $X$. We have (compare with Corollary 3 ):

Corollary 6. Let $\sigma$ be as above and assume that

$$
\inf _{C \cap M}\left(\sum_{t \in T} \lambda_{t} f_{t}\right) \leq 0 \text { for any } \lambda \in \mathbb{R}_{+}^{(T)} .
$$

Then $\sigma$ is consistent if and only if

$$
\bigcup_{x \in C \cap M}\left\{\left(\left(f_{t}(x)\right)_{t \in T}, 0\right)\right\}+\mathbb{R}_{+}^{T} \times \mathbb{R}_{+}
$$

is closed regarding $\left\{0_{T}\right\} \times \mathbb{R}$.

Proof: Apply Theorem 3 with $f \equiv 0$ and $\mu=0_{T}$. Observe that (5.2) is satisfied (with $\bar{\lambda}=0_{T}$ ) and that (5.3) amounts to $\sup \left(Q^{\mu}\right)=0$. Then it suffices to notice that $\min \left(P^{\mu}\right)=0$ amounts to say that $\sigma$ is consistent.

\section{Linear infinite PROBlems}

In this section we will apply the previous results, essentially Theorems 1, 2 and 3 , to the linear infinite problem

$$
(P) \inf _{x}\left\langle c^{*}, x\right\rangle \text {, s.t. } x \in C,\left\langle x_{t}^{*}, x\right\rangle \leq r_{t}, t \in T,
$$

where $\left(x_{t}^{*}, r_{t}\right) \in X^{*} \times \mathbb{R}, t \in T, c^{*} \in X^{*}$, and $C$ is a closed convex cone in the locally convex separate tvs $X$. One has straightforwardly,

$$
(D) \equiv(Q) \sup _{\lambda}-\left(\mathrm{i}_{C^{+}}\left(c^{*}+\sum_{t \in T} \lambda_{t} x_{t}^{*}\right)+\sum_{t \in T} \lambda_{t} r_{t}\right) \text {, s.t. } \lambda \in \mathbb{R}_{+}^{(T)} \text {. }
$$


Modifying the feasible set (but not the value) of $(D)$ we get a classical Haar dual-type problem

$$
\left(D^{\#}\right) \sup _{\lambda}-\sum_{t \in T} \lambda_{t} r_{t} \text {, s.t. } \lambda \in \mathbb{R}_{+}^{(T)}, \sum_{t \in T} \lambda_{t} x_{t}^{*} \in C^{+}-c^{*} .
$$

In order to apply Theorem 1 to the present situation, let us introduce the $w^{*}$-continuous linear mapping

$$
\Lambda: \mathbb{R}^{(T)} \rightarrow X^{*} \times \mathbb{R}, \Lambda(\lambda)=\sum_{t \in T} \lambda_{t}\left(x_{t}^{*}, r_{t}\right)
$$

Denoting by $K$ the characteristic cone of $\sigma:=\left\{\left\langle x_{t}^{*}, x\right\rangle \leq r_{t}, t \in T, x \in C\right\}$, one has

$$
\begin{aligned}
K & =\operatorname{epi}\left(\mathrm{i}_{C}^{*}\right)+\text { cone }\left(\bigcup_{t \in T} \operatorname{epi}\left(x_{t}^{*}-r_{t}\right)^{*}\right) \\
& =C^{-} \times \mathbb{R}_{+}+\operatorname{cone}\left(\bigcup_{t \in T} \operatorname{epi}\left(\mathrm{i}_{\left\{x_{t}^{*}\right\}}+r_{t}\right)\right) \\
& =C^{-} \times \mathbb{R}_{+}+\Lambda\left(\mathbb{R}_{+}^{(T)}\right)+\left\{0_{X^{*}}\right\} \times \mathbb{R}_{+} \\
& =C^{-} \times \mathbb{R}_{+}+\Lambda\left(\mathbb{R}_{+}^{(T)}\right) .
\end{aligned}
$$

Corollary 7. Assume that $(P)$ is consistent. Then, the following statements are equivalent:

(i) $\sup \left(D^{\#}\right)=-\infty$ or $\inf (P)=\max \left(D^{\#}\right) \in \mathbb{R}$.

(ii) $K$ is $w^{*}$-closed regarding to $\left\{-c^{*}\right\} \times \mathbb{R}$.

Proof: Theorem 1 establishes that $(i)$ holds if and only if $\mathfrak{B}$ is $w^{*}$-closed with respet to $\left\{0_{X^{*}}\right\} \times \mathbb{R}$. In this linear setting, we get straightforwardly, for any $\lambda \in \mathbb{R}_{+}^{(T)}$,

$$
\operatorname{epi}\left(\mathrm{i}_{C}+c^{*}+\sum_{t \in T} \lambda_{t}\left(x_{t}^{*}-r_{t}\right)\right)^{*}=\left(c^{*}, 0\right)+\Lambda(\lambda)+C^{-} \times \mathbb{R}_{+} .
$$

Consequently,

$$
\mathfrak{B}=\left(c^{*}, 0\right)+\Lambda\left(\mathbb{R}_{+}^{(T)}\right)+C^{-} \times \mathbb{R}_{+}=\left(c^{*}, 0\right)+K,
$$

and $\mathfrak{B}$ is $w^{*}$-closed regarding to $\left\{0_{X^{*}}\right\} \times \mathbb{R}$ if and only if $(i i)$ holds.

Remark 7. Whenever $(P)$ and $\left(D^{\#}\right)$ are both consistent, condition $(i i)$ in Corollary 7 characterizes the identity $\inf (P)=\max \left(D^{\#}\right)$ with the common value in $\mathbb{R}$.

Remark 8. According to the assumptions of Theorem 3, the convex cone $C$ does not need to be closed in Corollary 7 . 
We will now apply Theorem 3 for $\mu=0_{T}$ to the linear infinite problem $(P)$. To this end, let us consider the continuous linear mapping

$$
L: X \rightarrow \mathbb{R}^{T} \times \mathbb{R}, L(x)=\left(\left(\left\langle x_{t}^{*}, x\right\rangle\right)_{t \in T},\left\langle c^{*}, x\right\rangle\right) .
$$

We have (compare with [7, Theorem 5.5]):

Corollary 8. Assume that $c^{*} \in C^{+}-$cone $\left\{x_{t}^{*}, t \in T\right\}$. Then, the following statements are equivalent:

(i) $\sup \left(D^{\#}\right)=+\infty$ or $\min (P)=\sup \left(D^{\#}\right) \in \mathbb{R}$.

(ii) $L(C)+\mathbb{R}_{+}^{T} \times \mathbb{R}_{+}$is closed regarding to $\left\{\left(r_{t}\right)_{t \in T}\right\} \times \mathbb{R}$.

Proof: Applying Theorem 3 we observe that (5.2) is equivalent to $c^{*} \in$ $C^{+}-$cone $\left\{x_{t}^{*}, t \in T\right\}$, and we have

$$
E=L(C)+\mathbb{R}_{+}^{T} \times \mathbb{R}_{+}-\left\{\left(r_{t}\right)_{t \in T}\right\} \times\{0\} .
$$

Consequently, $E$ is closed regarding to $\left\{0_{T}\right\} \times \mathbb{R}$ amounts to statement (ii) in Corollary 7, and we are done.

Acknowledgement 1. The authors wish to thank the two anonymous referees for their valuable comments and suggestions that have significantly improved the quality of the paper.

Acknowledgement 2. M.A. Goberna and M.A. López were partially supported by MINECO of Spain, grant MTM2011-29064-C03-02.

\section{REFERENCES}

[1] Boț, R.I.: Conjugate duality in convex optimization. Springer-Verlag, Berlin/Heidelberg (2010)

[2] Dinh, N., Goberna, M.A., López, M.A.: From linear to convex systems: consistency, Farkas Lemma and applications. J. Convex Analysis 13, 279-290 (2006)

[3] Dinh, N., Goberna, M.A., López, M.A., Son, T.Q.: New Farkas-type constraint qualifications in convex infinite programming, ESAIM: Control, Optim. \& Calculus of Variations 13, 580-597 (2007)

[4] Ernst, E., Volle, M.: Zero duality gap for convex programs: a generalization of the Clark-Duffin Theorem. J. Optim. Theory Appl. 158, 668-686 (2013)

[5] Fang, D.H., Li, C., Ng, K.F.: Constraint qualifications for extended Farkas's lemmas and Lagrangian dualities in convex infinite programming. SIAM J. Optim. 20, 1311$1332(2009)$

[6] Goberna, M.A., López, M.A.: Linear semi-infinite optimization. J. Wiley, Chichester, U.K., (1998)

[7] Goberna, M.A., López, M.A., Volle, M.: Primal attainment in convex infinite optimization duality. J. Convex Anal. 21, to appear.

[8] Jeyakumar, V., Wolkowicz, H.: Zero duality gaps in infinite dimensional programming. J. Optim. Theory Appl. 67, 87-108 (1990)

[9] Joly, J.L.: Une famille de topologies et de convergences sur l'ensemble des fonctionnelles convexes, PhD Thesis, IMAG - Institut d'Informatique et de Mathématiques Appliquées de Grenoble (1970) 
[10] Joly, J.L., Laurent, P.J.: Stability and duality in convex minimization problems. Rev. Française Informat. Recherche Opérationnelle 5, 3-42 (1971)

[11] Laurent, P.-J. : Approximation et optimization (French). Hermann, Paris (1972)

[12] Moussaoui, M., Volle, M.: Quasicontinuity and united functions in convex duality theory. Comm. Appl. Nonlinear Anal. 4, 73-89 (1997)

[13] Pomerol, J.Ch.: Contribution à la programmation mathématique: existence de multiplicateurs de Lagrange et stabilité, PhD Thesis, Paris 6 (1980)

[14] Rockafellar, R.T.: Convex analysis. Princeton University Press, Princeton, N.J. (1970)

[15] Zălinescu, C.: Convex analysis in general vector spaces. World Scientific, River Edge, N.J. (2002)

Department of Statistics and Operations Research, University of Alicante, Alicante, Spain (mgoberna@ua.es), corresponding author.

Department of Statistics and Operations Research, University of Alicante, Alicante, Spain (marco.antonio@ua.es); And Federation University of Australia, Ballarat, Australia

Département de Mathématiques, Université D’Avignon, Avignon, France, (MICHEL.VOLLE@UNIV-AVIGNON.FR) 